\title{
Global diversity of stoneflies (Plecoptera; Insecta) in freshwater
}

\author{
Romolo Fochetti • \\ José Manuel Tierno de Figueroa
}

(C) Springer Science+Business Media B.V. 2007

\begin{abstract}
Plecoptera, or stoneflies, is a small order of hemimetabolous insects: according to our data, more than 3,497 species have been described so far in the world. The total number of species has enormously increased in the last 30 years $(2,000$ species estimated in 1976) and, if the trend continues, then it will nearly double in the near future. The order is divided into the suborders Arctoperlaria and Antarctoperlaria, and includes 16 families: 12 arctoperlarian and 4 antarctoperlarian. The Arctoperlaria account for a total number of 3,179 species, and Antarctoperlaria, only 318 species. The total number of genera is 286 . We give in this article the estimated number of species for each family. The fauna and diversity of stonefly in North America (650 species reported) and Europe (426 species) are best known. Nevertheless, in the last 25 years, a mean of 2.6 Plecoptera species per year were described in Europe. Stonefly-faunas of Australia (191 species, Tasmania included) and New
\end{abstract}

Guest editors: E.V. Balian, C. Lévêque, H. Segers \& K. Martens

Freshwater Animal Diversity Assessment

R. Fochetti ( $\square)$

Dipartimento di Scienze Ambientali, Università della Tuscia, Largo dell'Università snc, 01100 Viterbo, Italy

e-mail: fochetti@unitus.it

J. M. Tierno de Figueroa

Departamento de Biologia Animal, Facultad de Ciencias, Universidad de Granada, 18071 Granada, Spain
Zealand (104 species) are relatively well-known, while our knowledge of the Plecoptera of Central and South America (95 and 378 species respectively) is poor and still not representative of the real diversity. Africa has a reduced stonefly fauna (126 species). Asian stonefly diversity (approximately 1,527 species) is much greater than that of Europe or North America despite the fact that, except for Japan and Asiatic Russia that have been well studied, our knowledge of the remaining Asiatic areas is extremely poor. Even though our data indicate the Holarctic Region as the diversity hot-spot for the order, the analysis of the specific diversity divided by family suggests also an important role of tropical stoneflies.

Keywords Plecoptera · Distribution ·

Diversity $\cdot$ Zoogeographical regions

\section{Introduction}

Plecoptera is a small order of hemimetabolous insects, commonly called stoneflies, with more than 3,497 described species (this article). Stoneflies are distributed over all continents except Antarctica, and constitute a significant ecological component of running water ecosystems. Their ecological requirements greatly limit the dispersal capacity of the nymphs and, because adults have reduced flight ability, stoneflies show a high percentage of 